\title{
Small Bowel Evisceration Following Vaginal Hysterectomy is a General Surgical Emergency-A Case Report and Review of the Literature
}

\author{
Mandana Sigaroudinia, David Bowden, Deb Maitra, Graham Cawdell \\ Departments of General Surgery \& Gynaecology St Helens \& Knowsley NHS Trust Liverpool, \\ Merseyside, United Kingdom \\ E-mail: mandana.sigaroudinia@nhs.net
}

Received December 15, 2010; revised January 25, 2011; accepted March 11, 2011

\begin{abstract}
Small bowel evisceration through the vagina is a rare condition, which tends to affect post-menopausal women who have undergone vaginal hysterectomy. It is a surgical emergency with a favourable outcome if diagnosed and treated in a timely fashion. Delay in diagnosis can precipitate infarction of small bowel loops with associated morbidity and mortality. Treatment involves laparotomy, reduction of the incarcerated loops, plus/minus small bowel resection) and repair of the vaginal rupture. The purpose of this paper is to report a case of small bowel evisceration through the vagina, to review the literature pertaining to this topic and to raise awareness of this condition in the general surgical community.
\end{abstract}

Keywords: Small Bowel Evisceration, Vaginal Rupture, Hysterectomy

\section{Case Report}

A 67 year old lady underwent a vaginal hysterectomy in November 2008 and made an uneventful post operative recovery, being discharged on the $3^{\text {rd }}$ day. She was reviewed once in the outpatient department and subsequently followed up by her GP.

7 months later she presented to her GP complaining of a 'dragging' sensation in her vagina. She also described feeling a 'bubble' protruding through the vagina. She was not examined but was referred to the gynaecological outpatients department, at which time a vaginal prolapse was diagnosed. As a result she was given physiotherapy exercises as a means of treatment.

Over the following weeks she experienced increasing amounts of pain, which had become more stabbing in nature and was said to radiate to the peri-umbilical region. This pain was worse on walking, sitting down and on carrying out her exercises. She became reliant on regular analgesics.

She had no history of bleeding PV or PR and no change in her bowel habit. She had been passing some yellow jelly-like discharge and as such was using pads on her underwear.

6 weeks after being reviewed in the outpatient de- partment she was trying to pass urine when she felt faint and had a severe stabbing pain in the pelvic region. She noticed that she was bleeding and called an ambulance.

On examination in A\&E she was found to have 2 loops of small bowel protruding through the vagina. These were oedematous but viable. She underwent a laparotomy as a joint procedure between the gynaecologists and the general surgeons. At laparotomy approximately 1.5 feet of proximal ileum was protruding through a large defect in the posterior vaginal wall and vault. This was resulting from a wound dehiscence related to previous vaginal hysterectomy. The small bowel was reduced and the hernial sac was excised. The vaginal wall was repaired with vicryl and she was given 3 days of intravenous antibiotics. She made a good recovery and was discharged on the $4^{\text {th }}$ post-operative day.

\section{Discussion \& Literature Review}

The first reports of vaginal evisceration appear in the literature from C1950 as a complication of vaginal hysterectomy [1]. Over the ensuing years it is a condition which has been scantily reported in gynaecological journals most frequently in association with post menopausal women who have had a history of gynaecological surgery 


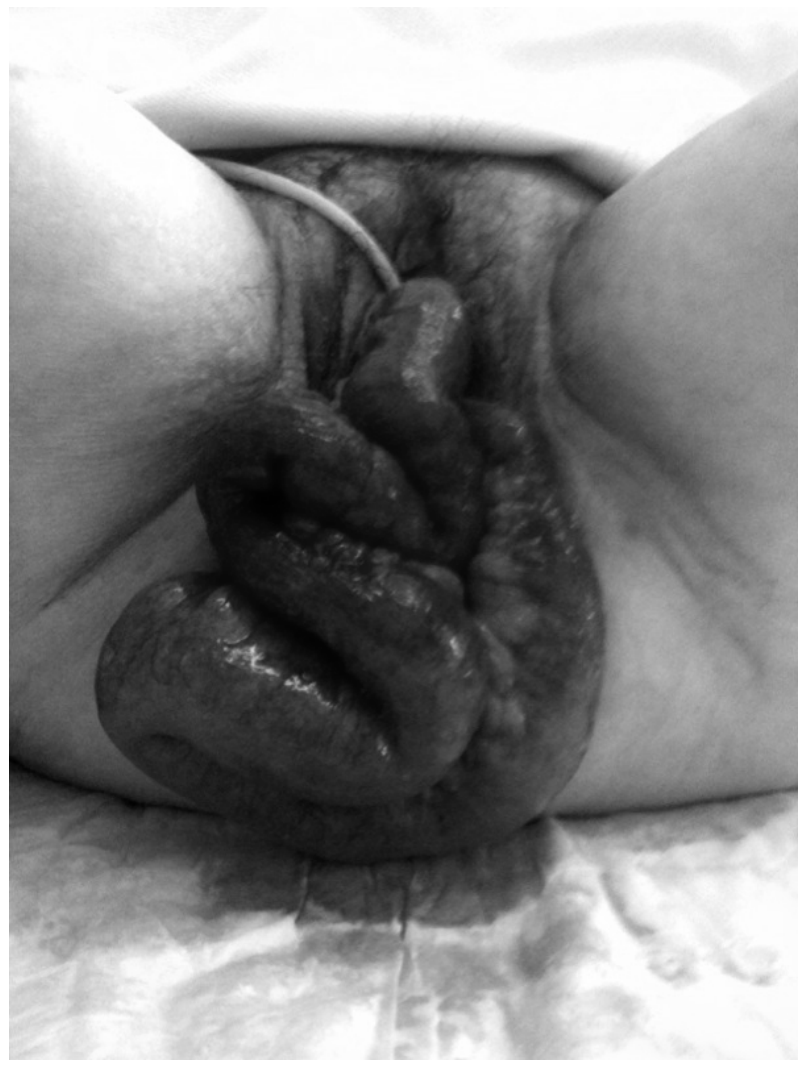

Figure 1. Small bowel loops eviscerating from the vagina.

or enterocoele [2]. It also tends to be associated with increased intra-abdominal pressure and conditions, which lead to weakened pelvic tissues [3]. The most common surgical precursor is the vaginal hysterectomy, which has been reported to account for over $60 \%$ of cases. Abdominal and laparoscopic hysterectomy may also contribute in approximately $30 \%$ and $5 \%$ of cases respectively [4]. Whilst vaginal evisceration is most common in the post-menopausal woman, it has been reported in the premenopausal lady who has had an abdominal hysterectomy, as a complication following coitus and posterior vaginal fornix rupture [5]. Other presentations, which are even more unusual, include following intra-peritoneal chemotherapy for ovarian cancer [6], evisceration secondary to water sports [7] or the use of vaginal brachytherapy [8].

Presenting features are vaginal bleeding or oozing, pelvic pain and a feeling of pressure in the abdomen and/or pelvis. The majority of cases find small bowel loops to be the eviscerating organ although greater omentum has also been described [9]. If the problem is not recognised early enough small bowel may incarcerate, requiring small bowel resection with its associated morbidity and mortality.

Surgery is the mainstay of treatment using a combined abdominal and vaginal approach, between the general surgeons and gynecologists [10]. More recently a pure vaginal approach has been described [11], as well as a combined vaginal and laparoscopic approach [12]. The small bowel is reduced and inspected for viability, the hernial sac is excised and the vaginal defect is repaired, usually with an absorbable suture such as Polyglactin or Polydiaxone. A small bowel resection may be performed in cases of bowel infarction. The patient is treated with broad-spectrum antibiotics.

Whilst in the majority of cases a primary repair of the vaginal defect is sufficient, some authors have reported using a mesh to reinforce the defect [13, 14]. Furthermore, techniques such as graciloplasty to reconstruct the rectovaginal septum and allogenic dermal grafting have also been described $[15,16]$.

The majority of cases report a favourable outcome if this condition is identified early and surgical intervention is prompt, highlighting the necessity for increased awareness about this condition in both the general surgical and gynaecological communities.

\subsection{Figure 1}

\section{References}

[1] C. E. Dunn, "Disruption of Vaginal Wound with Evisceration; Eighteen Months Following Vaginal Hysterectomy," Journal Of The Michigan State Medical Society, Vol. 49, No. 8, 1950, pp. 925-927.

[2] F. W. Kayser, N. S. Heaney and H.C. Dahleen, "Spontaneous Evisceration through Vaginal Enterocele,” American Journal of Obstetrics \& Gynecology, Vol. 61, No. 6, 1951, pp. 1393-1394.

[3] A. J. Croak, J. B. Gebhart, C. J. Klingele, G. Schroeder, "Characteristics of Patients with Vaginal Rupture and Evisceration,” Obstetrics \& Gynecology, Vol. 103, No. 3, 2004, pp. 572-576. doi:10.1097/01.AOG.0000115507.26155.45

[4] P. T. Ramirez, D. P. Klemer, "Vaginal Evisceration after Hysterectomy: a Literature Review," Obstetrical \& Gynecological Survey,Vol. 57, No. 7, 2002, pp. 462-467. doi:10.1097/00006254-200207000-00023

[5] K. Yuce, P. Dursun, M. Gultekin, "Postheyterectomy Intestinal Prolapse after Coitus and Vaginal Repair," Arch Gynecological Obstetrical, Vol. 272, No. 1, 2005, pp. 80-81. doi:10.1007/s00404-004-0709-8

[6] A. M. Burkett, D. E. Cohn, L. J. Copeland, "Vaginal Evisceration during Intraperitoneal Chemotherapy for Advanced Ovarian Cancer," Gynecologic Oncology, Vol. 104, No. 2, 2007, pp. 491-493. doi:10.1016/j.ygyno.2006.10.029

[7] J. L. Powell, M. B. Meyerson, "Vaginal Evisceration after Abdominal Hysterectomy and Vaginal Brachytherapy,” Journal of Pelvic Medicine and Surgery, Vol. 9, No. 3, 2003, pp. 125-128. doi:10.1097/01.SPV.0000074090.17386.38 
[8] Y. Avidor, R. Rub, Y. Kluger, "Vaginal Evisceration Resulting from a Water Slide Injury,” Journal Of Trauma Injury, Infection And Critical Care, Vol. 44, No. 2, 1998, pp. 415-416. doi:10.1097/00005373-199802000-00038

[9] W. D. Kang, S. M. Kin, H. S. Choi, "Vaginal Evisceration after Radical Hysterectomy and Adjuvant Radiation,” Journal of Gynecologic Oncology, Vol. 20, No. 1, 2009, pp. 63-64. doi:10.3802/jgo.2009.20.1.63

[10] A. A. Kambouris, B. H. Drukker, J. Barron, "Vaginal Evisceration. A Case Report and Brief Review of the Literature," Archives of Surgery, Vol. 116, No. 7, 1981, pp. 949-51.

[11] M. D. Moen, M. Desai, R. Sulkowski, "Vaginal Evisceration Managed by Transvaginal Bowel Resection and Vaginal Repair,” International Urogynecology Journal and Pelvic Floor Dysfunction, Vol. 14, No. 3, 2003, pp. 218-220. doi:10.1007/s00192-003-1056-1

[12] F. Narducci, Y. Sonoda, E. Lambaudie, E. Leblanc, "Vaginal Evisceration after Hysterectomy: the Repair by a Laparoscopic and Vaginal Approach with an Omental Flap,” Gynecologic Oncology, Vol. 89, No. 3, 2003, pp.
549-551. doi:10.1016/S0090-8258(03)00153-7

[13] D. Jurus, P. Finamore, B. Vakill, “Use of Synthetic Mesh to Prevent Recurrent Vaginal Evisceration: A Case Report," International Urogynecology Journal and Pelvic Floor Dysfunction, Vol. 20, No. 2, 2009, pp. 259-260. doi:10.1007/s00192-008-0699-3

[14] C. Crespi and A. M. De Giorgio, "Prolapse of the Vaginal Vault Complicated by Ileal Evisceration. Possible Role of Dacron Mesh in Emergencies," Gynecologic Chiropractic, Vol. 12, No. 10, 1991, pp. 498-500.

[15] V. Brehm, P. Steenvoorde, J. Oskam, "Vaginal Evisceration of Small Bowel Loops Following Prior Vaginal Hysterectomy: A Graciloplasty Performed to Reconstruct the Rectovaginal Septum,” International Journal of Surgery, Vol. 6, No. 6, 2008, pp. 34-35. doi:10.1016/j.ijsu.2006.11.004

[16] R. D. Moore, J. R. Miklos, "Repair of Vaginal Evisceration Following Colpocleisis Utilizing an Allogenic Dermal Graft," International Urogynecology Journal and Pelvic Floor Dysfunction, Vol. 12, No. 3, 2001, pp. 215217. doi:10.1007/PL00004035 